\title{
Cysts of the neuraxis of endodermal origin
}

\author{
I R A Mackenzie, J J Gilbert
}

Department of Pathology

(Neuropathology),

Victoria Hospital and

University of Western

Ontario, London,

Ontario, Canada

I R A Mackenzie

J J Gilbert

Correspondence to:

Dr Gilbert, Department of

Pathology (Neuropathology),

Victoria Hospital, South

Ontario N6A 4G5, Canada

Received 6 February 1990

and in revised form

18 May 1990.

Accepted 7 June 1990

\begin{abstract}
Five colloid cysts of the third ventricle were compared with two spinal enterogenous cysts to examine the hypothesis that these entities have the same origin from primitive endodermal tissue. All the lesions showed cuboidal and columnar epithelium with mucus containing goblet cells and cilia. Immunohistochemistry for cytokeratin, EMA and CEA was positive in all the colloid cyst and enterogenous cyst epithelium. S-100 was focally positive in three of the colloid and one of the enterogenous cysts while vimentin and GFAP were negative in both. The anatomical distribution of both colloid and enterogenous cysts is reviewed. An illustrative case of an identical cyst within the fourth ventricle is presented. This suggests that the similarities between colloid and enterogenous cysts and the presence of identical lesions along the neuroaxis indicate that these structures are derived from primitive foregut endoderm.
\end{abstract}

A variety of benign epithelial lined cysts of developmental origin may be found within the neuraxis. ${ }^{12}$ Some, such as dermoid, epidermoid and Rathke cleft cysts are known to arise from ectodermal tissue. ${ }^{2}$ Others such as epen- dymal cysts are clearly neuroectodermal in origin.

Colloid cysts of the third ventricle have had a variety of tissues of origin proposed, including primitive neuroepithelium, ${ }^{34}$ ependyma, ${ }^{5}$ choroid plexus epithelium ${ }^{34}$ and paraphyseal tissue. ${ }^{6}$ Based primarily on the ultrastructural appearance of the colloid cyst epithelium, a number of studies have suggested an alternative origin from primitive endodermal tissue..$^{7-9}$ If this latter hypothesis is true, then we would expect to see certain similarities between this lesion and another CNS cyst of known endodermal origin, such as, the spinal enterogenous cyst.

Spinal enterogenous cysts are usually found within the spinal canal at the cervicothoracic level in an intradural, extramedullary location. ${ }^{1}$ Their association with developmental defects of the overlying skin and vertebral bodies or fistulous connection with similar mediastinal, thoracic or abdominal cysts supports an origin from endodermal tissue. ${ }^{12}$

We compared colloid cysts of the third ventricle with enterogenous cysts of the spinal canal to either support or refute the hypothesis that these structures have a derivation in common from primitive endodermal tissue.

\section{Materials and methods}

Five surgically resected colloid cysts were
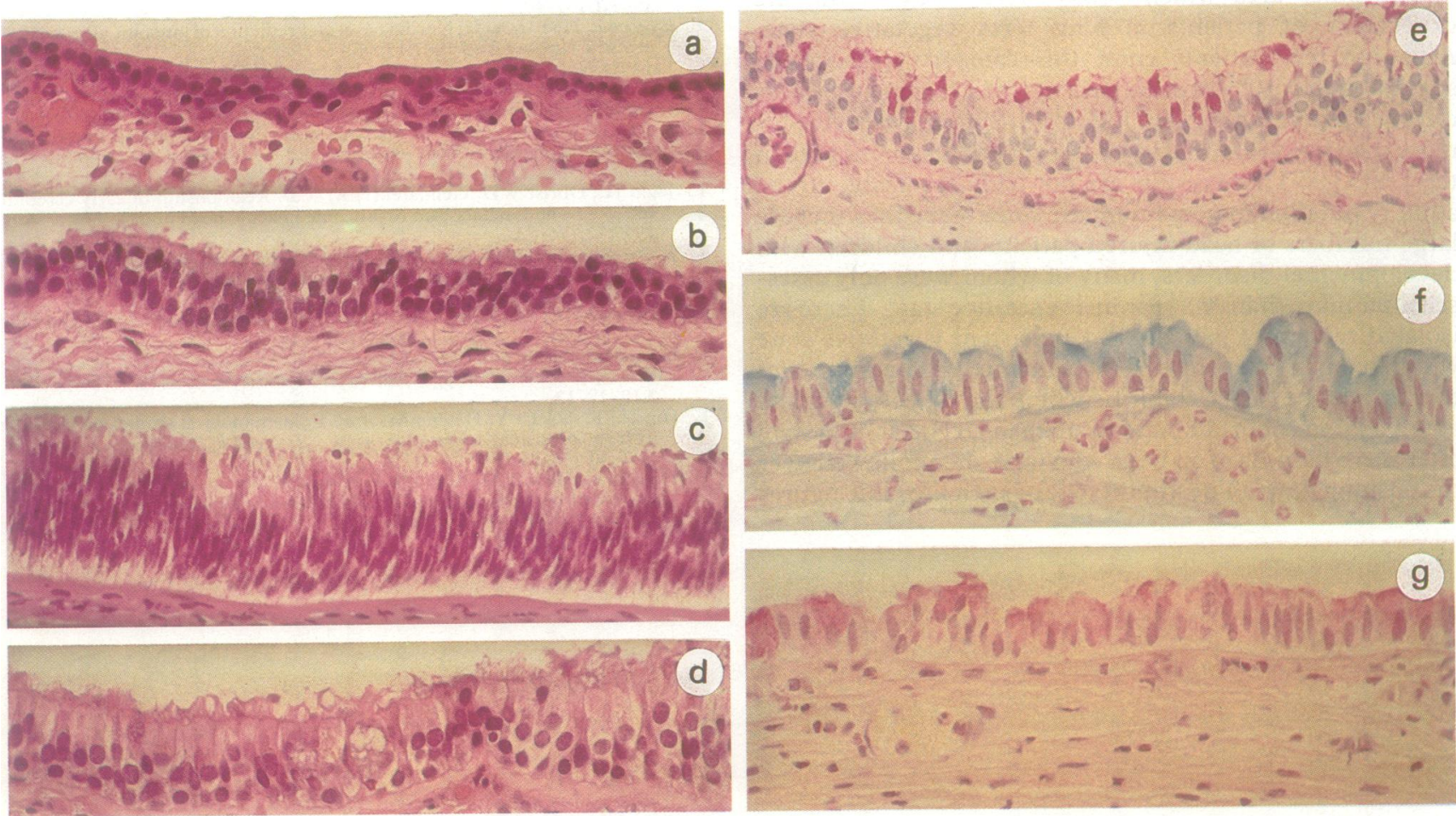

Figure 1 Colloid cyst epithelium: a) simple cuboidal, b) ciliated cells, c) tall columnar, d) ciliated columnar with goblet cells, e) mucus secreting, $P A S, f)$ Alcian Blue, $g)$ Mucicarmine. $(\times 80)$. 

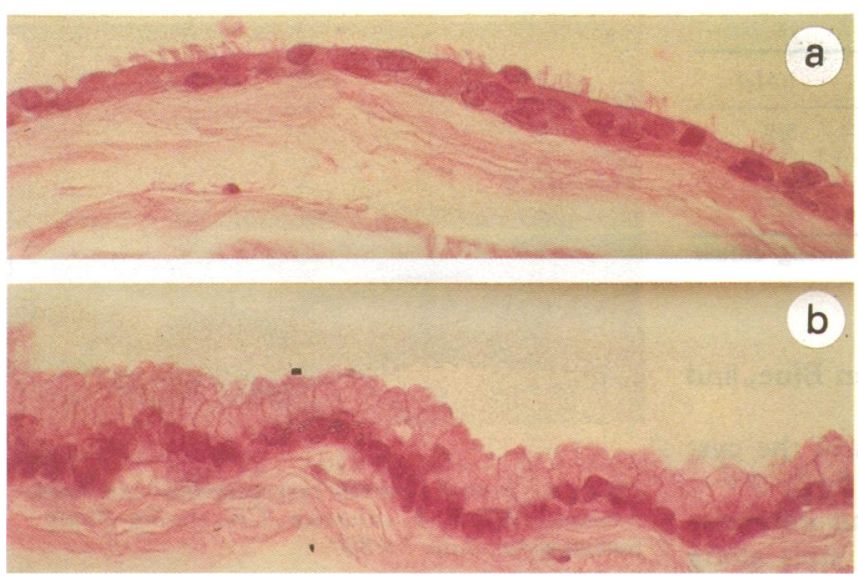

Figure 2 Enterogenous cyst epithelium: a) ciliated cuboidal, b) tall columnar epithelium, c) mucus secreting columnar PAS, d) Alcian Blue, e) Mucicarmine. $(\times 80)$.

obtained from three male and two female patients, ranging in age from 36 to 60 years of age (mean age, 45 years). Two enterogenous cysts were obtained from necropsies of male patients who presented with signs of spinal cord compression at ages 30 and 79 and died at 54 and 84 respectively. Available surgical examples of spinal enterogenous cysts did not contain an adequate amount of cyst epithelium for proper examination.

All tissue was formalin fixed, paraffin embedded and stained with hematoxylin and eosin, Masson's Trichrome, Periodic AcidSchiff, Alcian Blue, and Mucicarmine.

Immunohistochemical techniques were performed on formalin fixed paraffin embedded tissue using polyclonal antisera $(\mathbf{P})$ or monoclonal antibodies $(M)$ for tissue localisation of the following antigens (sources and dilutions in brackets): glial fibrillary acidic protein (GFAP, Sanbio 1:50, M), vimentin (VIM, Sanbio 1:50, M), cytokeratin (CK, Boehringer Mannheim 1:50, M), S-100 protein (S-100, Dako 1:5,000 P), epithelial membrane antigen
(EMA, Dako 1:50, M) and carcinoembryonic antigen (CEA, Dako 1:5,000 P). Standard Avidin-Biotin methods were employed using the Vectastain ABC kit (Vector Laboratories) with incubation for 18 hours in a sealed humidity chamber at room temperature and $\mathrm{AEC}$ as the chromogen.

\section{Results}

All five colloid cysts (fig 1) showed a fibrous capsule with an inner mucous secreting epithelial lining. In some regions, the epithelium appeared cuboidal with some cells having cilia while in other areas, columnar, with goblet cells. The epithelium stained positively with PAS, Alcian Blue, and Mucicarmine stains for mucus.

The two enterogenous cysts had a thin fibrous capsule and a simple epithelial lining. Both of the cysts showed primarily cuboidal epithelium with one also possessing areas of taller columnar epithelium and true goblet cells. Cilia were identified. The epithelium
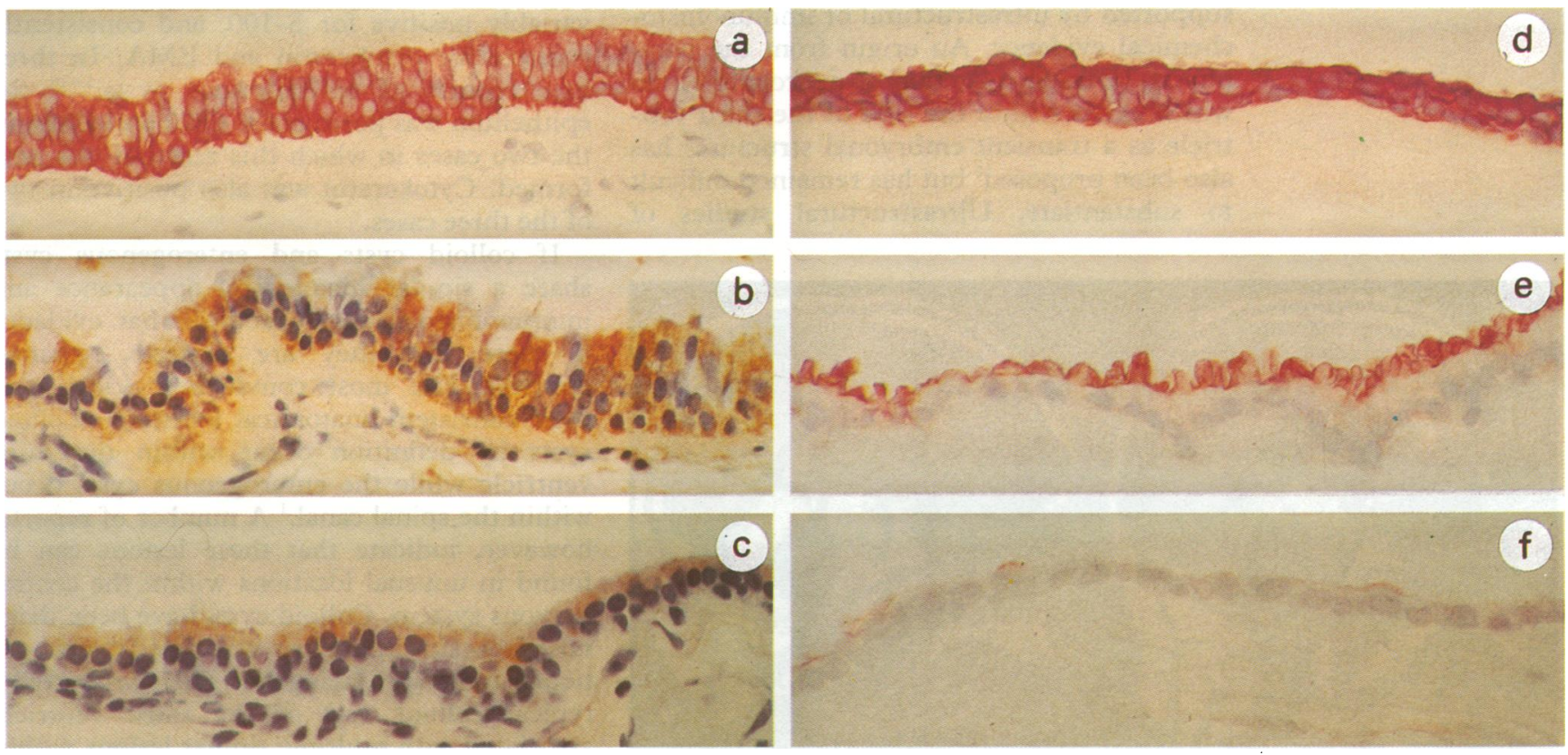

Figure 3 Immunohistochemistry of cyst epithelium. Colloid cyst epithelium: a) $C K, b) C E A, c)$ EMA. Enterogenous cyst epithelium: d) CK, e) CEA,f) EMA. Avidin Biotin immunoperoxidase. $(\times 80)$. 
Table Cyst immunohistochemistry

\begin{tabular}{lllllll}
\hline & GFAP & vimentin & $S-100$ & cytokeratin & CEA & EMA \\
\hline colloid cysts (5) & $0 / 5$ & $0 / 5$ & $3 / 5$ & $5 / 5$ & $5 / 5$ & $5 / 5$ \\
enterogenous cysts (2) & $(-)$ & $(-)$ & $(+/++)$ & $(+++)$ & $(++1+++)$ & $(+/++)$ \\
& $(-)$ & $0 / 2$ & $1 / 2$ & $2 / 2$ & $2 / 2$ & $2 / 2$ \\
& $(-)$ & $(+)$ & $(+++)$ & $(++)$ & $(+)$
\end{tabular}

KEY: negative $(-)$, weak focal positivity $(+)$, strong focal positivity $(++)$, strong diffuse positivity $(+++)$ stained positively with PAS, Alcian Blue, and Mucicarmine (fig 2).

Immunohistochemical reactions of the cyst epithelia are given in the table and fig 3.

Lesions of identical histology and immunocytochemistry are found in other sites along the neuroaxis.

A 60 year old man with three months of headache and unsteady gait, had papilloedema and a non-tender soft tissue mass on the back of the neck. The MRI (fig 4) showed a cystic lesion within the fourth ventricle and hydrocephalus of the lateral ventricles. The subcutaneous mass had the density of adipose tissue. No associated bony defect was seen. At surgery the neck mass was a lipoma, and the fourth ventricular cyst was biopsied and drained. The cyst epithelium (fig 5) showed areas of cuboidal and columnar epithelium, which stained positively with PAS, Alcian Blue, and Mucicarmine stains. Immunohistochemistry showed the epithelium to be strongly positive for cytokeratin, CEA, and EMA while completely negative for GFAP, vimentin, and S-100 protein.

\section{Discussion \\ Colloid cysts of the third ventricle have been somewhat enigmatic and despite their typical anatomic localisation to the anterior superior third ventricle and consistent morphological appearance, the exact tissue of origin has remained in debate. An origin from either ependymal or choroid plexus epithelium is not supported by ultrastructural or immunohisto- chemical evidence. An origin from the para- physis, (a small collection of neuroepithelium which exists within the roof of the third ven- tricle as a transient embryonal structure) has also been proposed ${ }^{6}$ but has remained difficult to substantiate. Ultrastructural studies of}

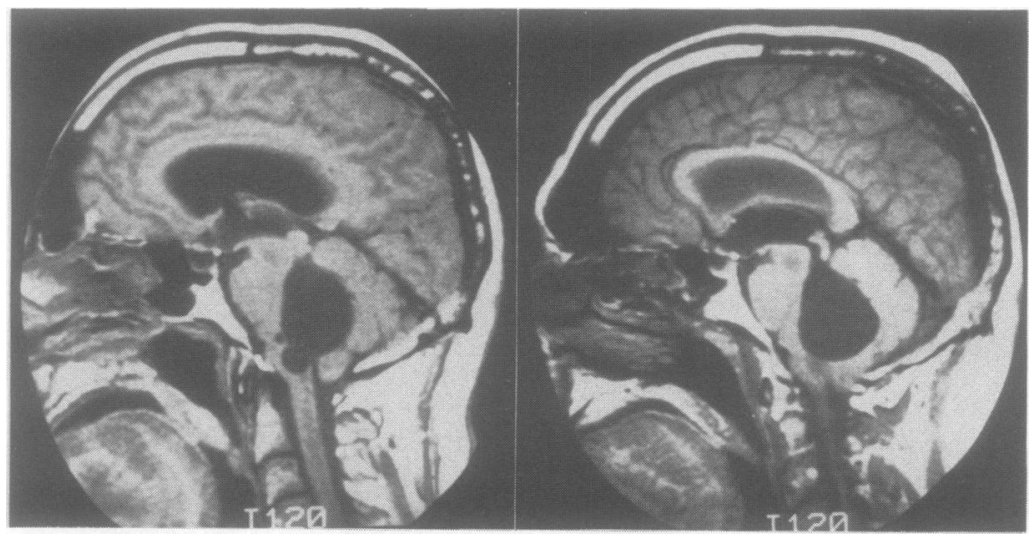

Figure 4 Sagittal T2 weighted MRI showing multilobular cyst expanding fourth ventricle and hydrocephalus of lateral ventricles.

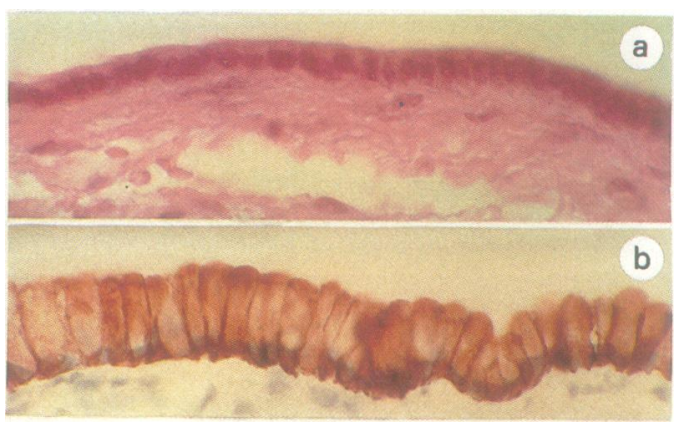

Figure 5 Fourth ventricle cyst epithelium: a) simple cuboidal, (HEE) b) columnar, CK, Avidin-Biotin immunoperoxidase. $(\times 80)$.

colloid cysts have shown some similarity with endodermal tissue and an origin from primitive foregut endoderm has been proposed. ${ }^{7-9}$ It is this latter suggestion that we have examined by comparing the colloid cyst structure and immunohistochemical profile with the spinal enterogenous (neurenteric) cyst.

We found striking similarities between the structure of both colloid and spinal enterogenous cysts; both possessing a simple mucus secreting epithelium which varies from columnar to cuboidal and occasionally possesses cilia and goblet cells. The immunohistochemical profile of the cyst epithelia is also remarkably similar with both lesions showing consistent positivity for cytokeratin, epithelial membrane antigen and carcinoembryonic antigen. This last marker, (CEA) while not specific, is often used as a marker for gut epithelium. ${ }^{10}$ Also, none of the lesions showed positivity for either GFAP or vimentin and in both groups of lesions there were occasional focal areas of epithelium which were weakly positive for S-100.

In two previous studies of colloid cysts, ${ }^{11} 12$ a total of 13 examples were examined and found to be negative for GFAP and vimentin, variably positive for $S-100$, and consistently positive for cytokeratin and EMA. In three case reports of enterogenous cysts, ${ }^{1-15}$ the epithelium was positive for CEA and EMA in the two cases in which this reaction was performed. Cytokeratin was also positive in two of the three cases.

If colloid cysts and enterogenous cysts share a similar histological appearance and immunohistochemical profile, what evidence is there that they are discrete, separate entities? The most convincing evidence is their consistent anatomical locations. Colloid cysts by definition occur within the third ventricle while the enterogenous cysts occur within the spinal canal. ${ }^{1}$ A number of reports, however, indicate that these lesions can be found in unusual locations within the central nervous system. Colloid cysts have been diagnosed outside the third ventricle. ${ }^{316}$ Of particular interest, Shuangshoti (1977) reported a typical colloid cyst of the third ventricle associated with multiple similar lesions within the supratentorial subarachnoid space. ${ }^{17}$ Spinal enterogenous cysts have been reported 
in a variety of locations outside the spinal canal including the infratentorial subarachnoid space, ${ }^{18-20}$ the fourth ventricle, ${ }^{21}$ and within the brain stem parenchyma. ${ }^{1322}$ Walls (1985) reported one patient with multiple enterogenous cysts within the lateral ventricle, fourth ventricle, and cerebral parenchyma. ${ }^{15}$ In the patient illustrated, the cyst was present in the fourth ventricle.

These cases in which colloid and enterogenous cysts have been diagnosed in unusual locations along the neuroaxis makes the diagnostic significance of specific anatomical locations less certain. The morphological and immunohistochemical similarities between colloid and enterogenous cysts combined with previous ultrastructural studies suggesting similarities of colloid cyst with endodermal tissue, suggest to us that these lesions are all derived from primitive foregut endoderm.

Presented in part at the 29th Annual Meeting of the Canadian Association of Neuropathologists, Montreal, Quebec, September 1989.

We thank the Pathology Department at University Hospital in London, Ontario, for contributing material and Miss Lynn James for her help in preparing this manuscript.

1 Russell DS, Rubinstein LJ. Pathology of tumours of the nervous system, 5th ed. Edition 5, London: Edward Arnold, 1989:704-6, 721-25.

2 Leech RW, Olafson RA. Epithelial cysts of the neuraxis. Arch Pathol Lab Med 1977;101:196-202.

3 Shuangshoti S, Netsky MG. Neuroepithelial (colloid) cysts of the nervous system. Further observation on pathogenesis, location, incidence and histochemistry. Neurol 1966;16:887-903.

4 Shuangshoti A, Roberts MP, Netsky MG. Neuroepithelial (colloid) cysts. Arch Path 1965;80:214-24.
5 Coxe WS, Luse SA. Colloid cyst of the third ventricle. An electron microscopic study. J Neuropathol Exper Neurol 1964;23:431-44.

6 Kappess JA. The development of the paraphysis cerebri in man with comments on its relationship to the intercolumnar tubercle and its significance for the origin of cystic nar tubercle and its significance for the origin of cystic tumours in

7 Ghatak NR, Kasoff I, Alexander E. Further observations on the fine structure of a colloid cyst of the third ventricle. Acta Neuropathol 1977;39:101-7.

8 Hirano A, Ghatak NR. The fine structure of colloid cysts of the third ventricle. $J$ Neuropathol Exper Neurol 1974;33: 333-41.

9 Yagishita S, Itoh Y, Shiozawa T, Tanaka T. Ultrastructural observation on a colloid cyst of the third ventricle. Acta Neuropathol (Berl) 1984;65:41-5.

10 Nadji M, Morales AR. Immunoperoxidase techniques: a practical approach to tumour diagnosis. Am Soc Clin Path, Chicago 1986.

11 Kondziolka D, Bilbao JM. An immunohistochemical study of neuroepithelial (colloid) cysts: primary lesions of neuroectodermal development. CANP 28th Annual Meeting, Toronto, Ontario. 1988.

12 Shibata T, Burger PC, Kleihues P. Origin of colloid cyst: immunoperoxidase study. No To Shinkei 1987;39:953-8.
immuno

13 Lach B, Russell N, Atack D, Benoit B. Intraparenchymal epithelial (enterogenous) cyst of the medulla oblongata. Can J Neurol Sci 1989;16:206-10.

14 Miyagi K, Mukawa J, Mekaru S, Ishikawa Y, Kinjo T Nakasone S. Enterogenous cysts in the cervical spinal canal. J Neurosurg 1988;68:292-6.

15 Walls TJ, Purohit DP, Aji WS, Schofield IS, Barwick DD. Multiple intracranial enterogenous cysts. $J$ Neurol Neurosurg Psychiatry 1986;49:438-41.

16 Parkinson D, Childe AE. Colloid cyst of the fourth ventricle. Report of a case of two colloid cysts of the fourth ventricle. J Neurosurg 1952;9:404-9.

17 Shuangshoti S, Phisitbutr M, Kasantikul V, Netsky M Multiple neuroepithelial (colloid) cysts: association with other congenital anomalies. Neurol 1977;27:561-6.

18 Chavda SV, Davies AM, Cassar-Pullicino VN. Enterogenous cysts of the central nervous system: a report of eight cases. Clin Radiol 1985;36:245-51.

19 Giombini S, Lodrini S, Migliavacca F. Intracranial enterogenous cyst. Surg Neurol 1981;16:271-3.

20 Hirai O, Dawamura J, Fukumitsu T. Prepontine epithelium-lined cyst. $J$ Neurosurg 1981;55:312-7.

21 Afshar F, Scholtz CL. Enterogenous cyst of the fourth ventricle. Case report. J Neurosurg 1981;54:836-8.

22 Zalatnai A. Neuroenteric cyst of medulla oblongata-a curiosity. Neuropediat 1987;18:40-1.

\section{Neurological stamp}

\section{Albucasis or Abul Kasim (936-1013)}

Albucasis, a distinguished Spanish Arabian physician and native of Cordoba, did much to raise the status of surgery which "had passed into the hands of vulgar and uncultivated minds and had fallen into contempt". His chief work was the Collection, or Tasrif, and was a complete account of surgery and medicine. He recommended the use of a red hot piece of iron for intractable migraine and cautery for apoplexy and epilepsy. He knew that fracture of the spine could result in paralysis.

This Syrian stamp was issued in 1964 to commemorate the Fourth Arab Dental and Oral Surgery Congress in Damascus. Albucasis was apparently the first to write on the treatment of deformities of the mouth and dental arches. (Stanley Gibbons 850, Scott C314).
الجمهروبلةالمربية SYRIAN ARAB REPUBLIC

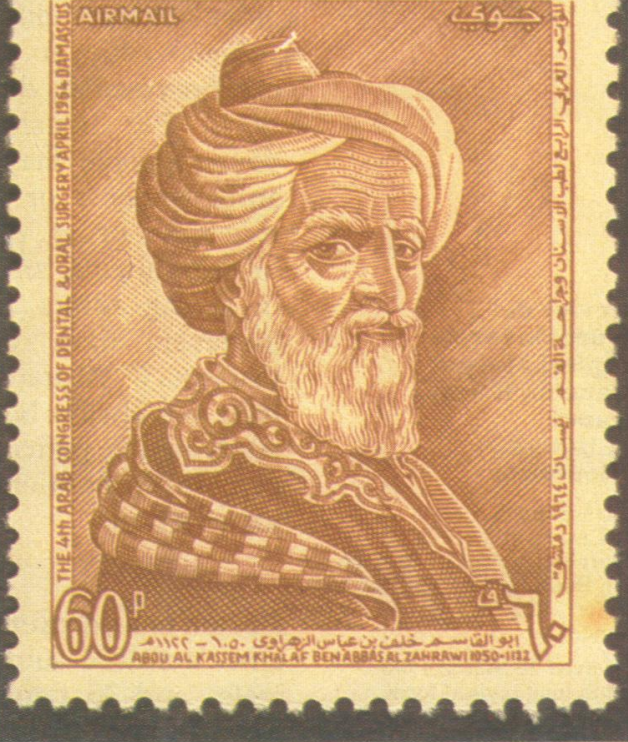

